\title{
Influence of atomic oxygen irradiation during deposition on crystallization of post-annealed barium zirconate thin films
}

\author{
Fumitada Iguchi ${ }^{1 *}$, Yoshikazu Shibata ${ }^{1}$, Takamichi Miyazaki ${ }^{1}$, Noriko Sata ${ }^{2}$, and Hiroo Yugami ${ }^{1}$ \\ ${ }^{1}$ Graduate School of Engineering, Tohoku University, Sendai 980-8579, Japan \\ ${ }^{2}$ Institute of Technical Thermodynamics, German Aerospace Center (DLR), Pfaffenwaldring 38-40, 70569 Stuttgart, Germany \\ E-mail: f_iguchi@energy.mech.tohoku.ac.jp \\ Received July 10, 2014; accepted August 28, 2014; published online October 15, 2014
}

The role of atomic oxygen irradiation in the epitaxial crystallization of yttrium-doped barium zirconate thin films fabricated by pulsed laser deposition (PLD) was investigated. X-ray diffraction and transmission electron microscopy revealed that, for films deposited without irradiation, random nucleation and growth occurred below the onset temperature for continuous crystallization at the film-interlayer interface. In contrast, for films deposited with oxygen irradiation, random nucleation and growth was not detected at the temperature of continuous crystallization, which facilitates epitaxial crystallization in these films. This study suggests the combined low temperature deposition with atomic oxygen irradiation and postannealing could control microstructure of solid-state electrochemical devices such as solid oxide fuel cells and solid-state lithium secondary batteries. (C) 2014 The Japan Society of Applied Physics

\section{Introduction}

Oxide layers deposited on substrates, with thicknesses varying between several tens and hundreds of nanometers, are extensively used in the industry for various applications such as semiconductors, superconductors, optical and photonics devices, and energy conversion systems. Several techniques, such as solidification, chemical vapor deposition, and other physical vapor deposition techniques, which include molecular beam epitaxy and vacuum deposition, have been developed to grow these oxide thin films. Among these techniques, plasma vapor deposition (PVD) presents advantages in material flexibility over these techniques because it relies on high energy sputtering, laser, and electron-beam to produce plasma vapors. ${ }^{1-7)}$

In the field of solid electrochemical devices, such as solidstate lithium secondary batteries and solid oxide fuel cells, PVD has attracted considerable attention of many researchers, because it can be used to easily deposit fully dense oxide thin films at temperatures lower than conventional sintering temperatures, thus avoiding significant elemental diffusion or production of a secondary phase. ${ }^{8-15)}$ Moreover, PVD can provide single crystal-like (epitaxial) films that are highly oriented in the vertical and lateral directions to substrates with columnar structures, although high temperature deposition is required to allow adsorbed materials to diffuse along substrate surfaces. ${ }^{16-22)}$ These epitaxial films are used for fundamental research, which clarifies the influence of lattice plane into electrical properties in the field of solid-state electrochemistry. ${ }^{16,18,20)}$

Solid-phase epitaxy techniques combining low-temperature deposition with post-annealing have also been explored for producing epitaxial thin films except for this field. However, related oxides were reported to be grown epitaxially by these techniques. For example, an epitaxially grown $\mathrm{SrTiO}_{3}$ (STO) thin film was obtained on a Si substrate using electron beam deposition followed by post-annealing at $650{ }^{\circ} \mathrm{C} .^{23)}$ Complex-layered $\mathrm{InGaO}_{3}(\mathrm{ZnO})_{\mathrm{m}}$ ( $\mathrm{m}$ is an integer) oxides deposited on a yttria stabilized zirconia (YSZ) single crystal substrate by pulsed laser deposition (PLD) epitaxially grew upon post-annealing at $1400^{\circ} \mathrm{C} .{ }^{24)}$ Other oxide epitaxial films, such as $\mathrm{YbGaO}_{3}(\mathrm{ZnO})_{5}, \mathrm{InGaO}_{3}(\mathrm{ZnO})_{5},{ }^{25)}$ and $\mathrm{YBa}_{2}-$ $\mathrm{Cu}_{3} \mathrm{O}_{7-x}(\mathrm{YBCO}),{ }^{26)}$ have been grown.
Recently, the epitaxial crystallization of perovskite-type high-temperature proton conductors such as yttrium-doped strontium zirconate and barium zirconate have been reported. ${ }^{27)}$ These proton conductors were deposited on $\mathrm{MgO}(100)$ or $\mathrm{STO}(100)$ single crystal substrates using PLD, and they were subjected to post-annealing in ambient air over $600{ }^{\circ} \mathrm{C}$. To the best of our knowledge, these are the first reports of a new type of solid-phase epitaxy in this field. Note that these films were synthesized under atomic oxygen irradiation without any interlayers at room temperature, and they still underwent epitaxial crystallization with annealing. If the atomic oxygen was not irradiated, the post annealed films became only polycrystalline.

Atomic oxygen (the oxygen radical), which exhibits higher reactivity compared to molecular oxygen, has been widely used to manufacture oxide thin films, especially superconductors such as yttrium barium copper oxide (YBCO). Previous reports have demonstrated that atomic oxygen irradiation enhances film oxidation and affects the epitaxial relationship between the film and substrate during hightemperature deposition. ${ }^{28,29)}$ However, the influence of atomic oxygen irradiation during deposition on the crystallization process at post-annealing has not been clarified. Because of high reactivity, it has been speculated that excess oxygen might be introduced in films and this chemical composition fluctuation possibly decreases activation energy for epitaxial growth. However, to date, there has been no experimental evidence exists to support this speculation.

If this type of growth could be used in thin film deposition in the field of solid-state electrochemistry, we could control microstructures of the thin films and obtain solid-state lithium secondary batteries and micro-solid oxide fuel cells with epitaxial electrolytes or electrodes, which could lead to revolutionary developments in electrochemical devices.

Hence, this study aims to experimentally clarify the role of atomic oxygen irradiation. Yttrium-doped barium zirconate (BZY) was selected as the target film material, and it was deposited by PLD with atomic oxygen irradiation on $\mathrm{SiO}_{2}$ glass substrates precoated with an STO interlayer. The purpose of precoating the STO interlayer was to keep thermal stress constant during post-annealing, as the composition of the interlayer changes. Because the feature of solid-phase epitaxy is continuous, epitaxial crystal growth from the inter- 


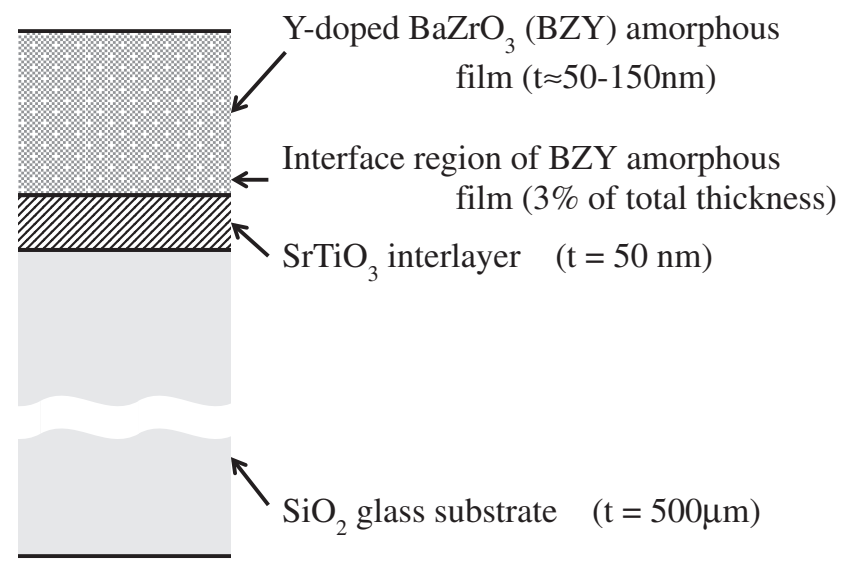

Fig. 1. Schematic illustration showing the structure of the prepared BZY thin films.

face between substrates and films, as well as the microstructure and crystal structure were examined by ex situ Xray diffraction (XRD) and transmission electron microscopy (TEM). Deposited films were post-annealed at various temperatures, and the effect of oxygen atomic irradiation on crystallization was evaluated.

\section{Experimental methods}

Polycrystalline pellets of BZY containing $5 \mathrm{~mol} \%$ yttrium were used as targets. These targets were synthesized by a solid-state reaction method as follows: $\mathrm{BaCO}_{3}(99.99 \%$, Rare Metallic), $\mathrm{ZrO}_{2}$ (Tosoh TZ-0), and $\mathrm{Y}_{2} \mathrm{O}_{3}$ (99.99\%, Nippon Yttrium) were mixed and calcined at $1200^{\circ} \mathrm{C}$ for $10 \mathrm{~h}$ in ambient air. The resultant powder was consolidated under isostatic pressure and sintered at $1600^{\circ} \mathrm{C}$ for $10 \mathrm{~h}$ in ambient air. Finally, the target pellet was obtained by mechanical polishing of the surface. Note that the STO single crystal (Furuuchi Chemical) was used as a target for precoating of the interlayer.

BZY thin films and STO interlayers were synthesized by PLD using an ArF excimer laser at a wavelength of $193 \mathrm{~nm}$ (Coherent COMPex 205). ${ }^{30)}$ First, the STO interlayer was deposited on a commercial grade $\mathrm{SiO}_{2}$ glass substrate at a substrate temperature of $800^{\circ} \mathrm{C}$ and an oxygen partial pressure of $0.3 \mathrm{~Pa}$. The BZY film deposition was conducted at room temperature and an oxygen partial pressure of $0.3 \mathrm{~Pa}$. The number of laser pulses was controlled to obtain a film thickness of $50-150 \mathrm{~nm}$. The laser frequency was set at $4 \mathrm{~Hz}$ and the energy was varied from 30 to $60 \mathrm{~mJ}$ for the BZY thin films and was set to $60 \mathrm{~mJ}$, which was measured in front of the laser irradiation window, for the STO interlayers.

Early stages of the BZY film deposition, corresponding to the first $3 \%$ of the total film thickness (the interface region of BZY in Fig. 1), were performed under atomic oxygen irradiation. Atomic oxygen was produced using a small helicon plasma source (ULVAC) at RF $150 \mathrm{~W}$ radiated toward the substrate through a small orifice (Fig. 2). On the surface of the substrate, the density of atomic oxygen was estimated to be $10^{15}$ atoms $/\left(\mathrm{cm}^{2} \mathrm{~s}\right)$. After deposition of the interface region, power supply to the plasma source was stopped, and then the deposition was continued. Control BZY specimens without atomic oxygen irradiation were also deposited for comparison.

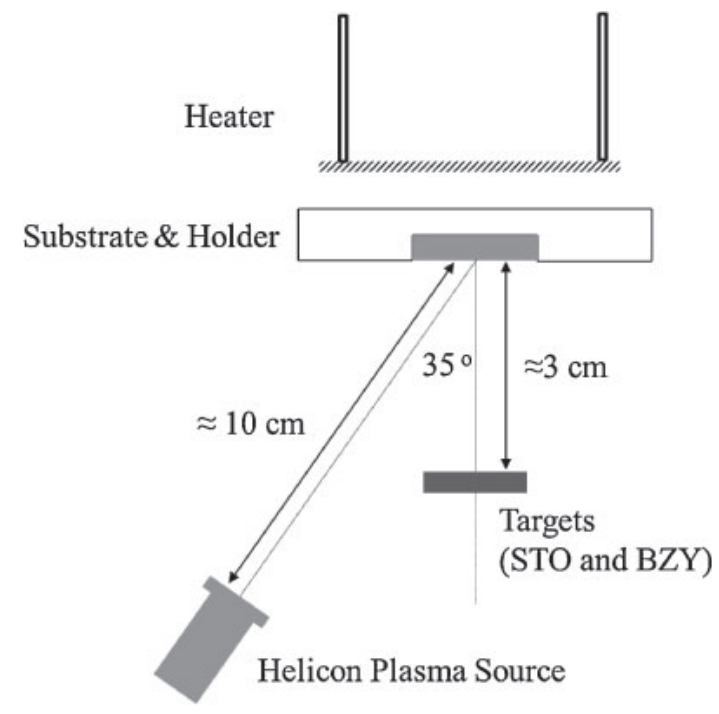

Fig. 2. Configuration of the substrate, the targets, and the helicon plasma source in the PLD process. These items are stored in a vacuum chamber.

The deposited BZY films were amorphous and were crystallized by post-annealing in ambient air at temperatures ranging from 150 to $750^{\circ} \mathrm{C}$ for up to $10 \mathrm{~h}$. Crystal structure and microscale morphology were examined in detail to evaluate the crystallization in post-annealed BZY films. Crystal structures were determined by XRD (PANalytical X'pert Pro) and microstructures were observed by TEM (Hitachi HF-2000).

\section{Results and discussion}

In this study, the STO interlayer was incorporated between the $\mathrm{SiO}_{2}$ substrate and the BZY film, because epitaxial growth has been reported for the $\operatorname{STO}(100)$ substrate. ${ }^{27)}$ Moreover, the STO interlayer prevented barium diffusion from the film to the substrate during post-annealing. The XRD pattern of the BZY amorphous film on the STO interlayer, as shown in Fig. 3(c), shows the STO interlayer was oriented along two planes, i.e., (110) and (100), probably caused by the difference of surface energy in each lattice plane. Generally, oxide thin films deposited at high temperature have a columnar structure, and this structure was also exhibited by the STO interlayer. Because each column has the same orientation, the (110) and (100) lattice planes are exposed on the surface of the STO interlayer; thus, BZY are deposited on these surfaces. The epitaxial relationship between BZY and STO was revealed to be "cube-on-cube". Thus, continuous epitaxial crystal growth of BZY thin films occurred on each column of STO. Therefore, the crystal structure of the BZY thin films would also be oriented along these directions. We recognized continuous crystallization of the BZY films from XRD patterns. Figures 3(a) and 3(b) show the XRD patterns of post-annealed BZY films deposited without and with atomic oxygen irradiation, respectively. Both patterns presented two diffraction peaks that were attributed to $\mathrm{STO}(110)$ and (200), along with a broad peak in the range of $20-30^{\circ}$, corresponding to the $\mathrm{SiO}_{2}$ substrate. The XRD pattern of an as-deposited amorphous film [Fig. 3(c)] did not exhibit any characteristic peaks for BZY; however, films post-annealed at $750{ }^{\circ} \mathrm{C}$ for $10 \mathrm{~h}$ 


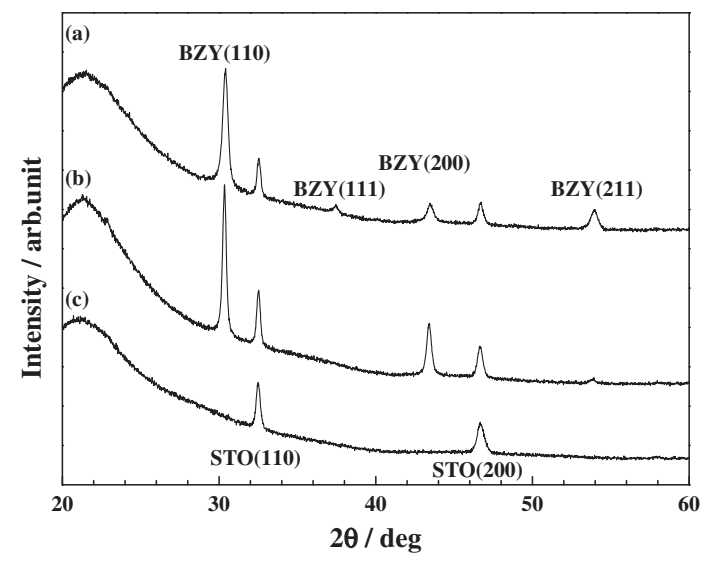

Fig. 3. XRD patterns of the BZY thin films. (a) Film deposited without atomic oxygen irradiation and crystallized by post-annealing at $750^{\circ} \mathrm{C}$ for $10 \mathrm{~h}$ in ambient air, (b) film deposited under atomic oxygen irradiation and crystallized by post-annealing at $750^{\circ} \mathrm{C}$ for $10 \mathrm{~h}$ in ambient air, and (c) asdeposited amorphous film. Films were deposited on an STO interlayer on a $\mathrm{SiO}_{2}$ substrate

showed some XRD peaks that can be attributed to the BZY film, evidence for crystallization. On the other hand, the film deposited under atomic oxygen irradiation showed fewer peaks than the one deposited without irradiation. Peaks corresponding to BZY(111) and (211) lattice planes were observed in the film deposited without irradiation. However, their intensities were significantly reduced in the film deposited under irradiation, resulting in the disappearance of the BZY(111) peak in this film. In contrast, the peak intensities for BZY(200) clearly increased in the irradiated film compared with the film deposited without irradiation, suggesting that atomic oxygen irradiation facilitates epitaxial crystallization.

Cross-sectional TEM observations were conducted to visualize the change in microstructure resulting from atomic oxygen irradiation (Fig. 4). The contrast was changed from the left- to the right-hand side of the image to enhance the image of the middle and bottom regions. Figure 4(a) shows the cross-sectional image of a BZY film deposited without irradiation and crystallized by post-annealing. On the lefthand side, small polygonal grains, which were several tens of nanometers in size, are seen in the middle of the BZY film. Moreover, the right-hand portion of the micrograph shows the presence of a BZY columnar structure localized near the interface between the BZY film and STO interlayer. In contrast, this columnar structure is visible throughout the entire BZY film deposited under irradiation [Fig. 4(b)]. The distribution of light spots observed in electron backscattering diffraction (EBSD) patterns indicated that the film deposited under irradiation was epitaxially oriented, whereas the film deposited without irradiation was polycrystalline.

These XRD and TEM results reveal that without irradiation the BZY film was polycrystalline by post-annealing. In contrast, when deposited under atomic oxygen irradiation, the crystallized film exhibited a columnar structure and adopted the same orientation as the STO interlayer, consistent with local epitaxy. The reported epitaxial growth was successfully reproduced in this study. ${ }^{27)}$ Comparing Figs. 4(a) and 4(b), the film thicknesses are different, because these films were deposited with laser energy of $30 \mathrm{~mJ}$ and deposition rate per
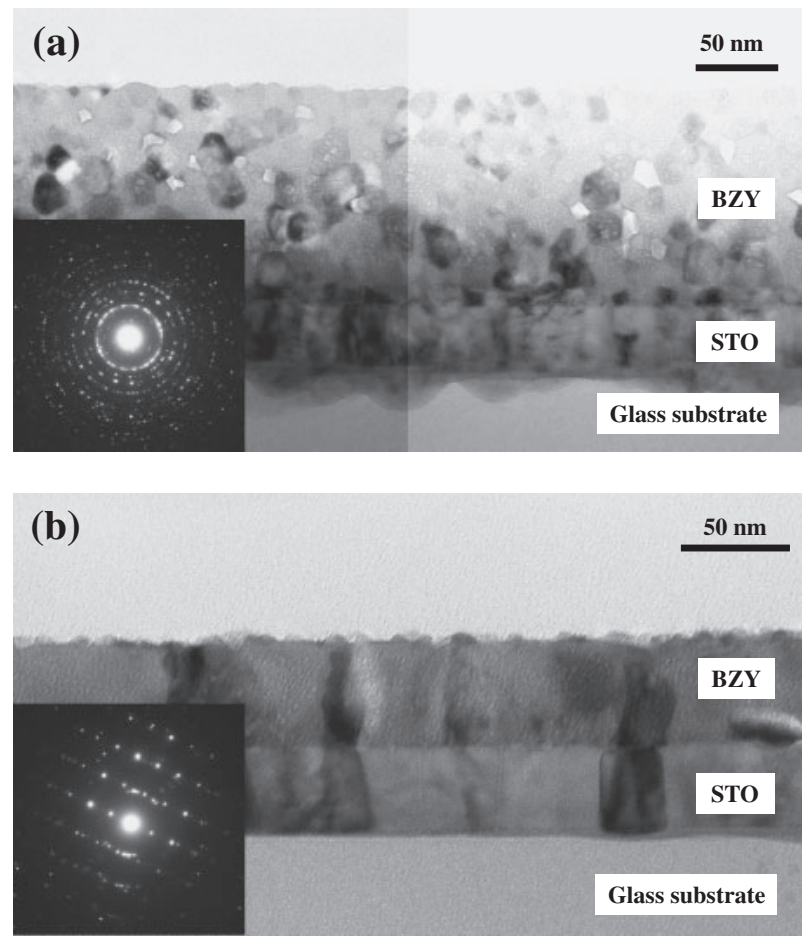

Fig. 4. Cross-sectional TEM images of crystallized BZY thin films. (a) Film deposited without atomic oxygen irradiation and crystallized by post-annealing at $750^{\circ} \mathrm{C}$ for $10 \mathrm{~h}$ in ambient air and (b) film deposited under atomic oxygen irradiation and crystallized by post-annealing at $750{ }^{\circ} \mathrm{C}$ for $10 \mathrm{~h}$ in ambient air. Insets show the EBSD patterns.

one laser pulse in such case fractured. However, the influence of atomic oxygen irradiation, as shown in Fig. 3, was similar in the deposited BZY films; therefore, we believed that the thickness of BZY films is not an essential property for the results.

Using these films, we can evaluate the role of atomic oxygen irradiation. Generally, annealed amorphous films are crystallized by thermally activated random nucleation and growth similar to glass, and the temperature at which crystallization starts depends on the size of the nanoscale seed in amorphous films. In contrast, solid-phase epitaxy occurs by continuous crystallization from the substrate-film interface to the film surface. Simultaneously, atoms in an amorphous film should move to each epitaxially predetermined position. Although that movement happens over an extremely short distance, it requires energy, and thus a higher temperature than thermally activated random nucleation. To solve this problem, very thin interlayers, such as $\mathrm{Sr}$ metal and $\mathrm{ZnO}$, are used. As shown in Fig. 4(a), the BZY film shows both a polycrystalline area in the middle of the BZY film and a columnar structure near the film-interlayer interface. This observation suggests that random nucleation and continuous crystallization was initiated at similar temperatures; however, continuous crystallization probably required a slightly higher temperature, and it was prevented by polygonal grains produced by random nucleation.

Combined with previous reports, ${ }^{23-26)}$ these results indicate that atomic oxygen irradiation during deposition may facilitate continuous crystallization at the film-interlayer interface and decrease the temperature at which crystallization starts. This irradiation may cause a nanoscale seed layer 


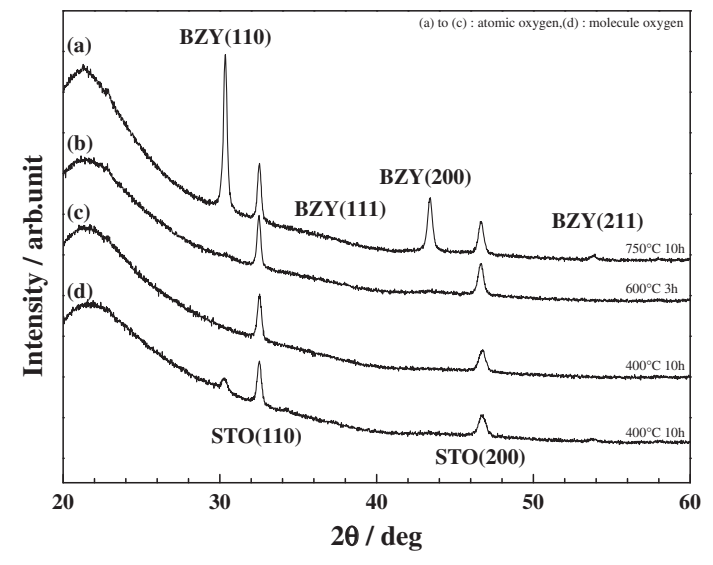

Fig. 5. XRD patterns of BZY thin films crystallized under different postannealing conditions. Films deposited under atomic oxygen irradiation and crystallized by post-annealing (a) at $750{ }^{\circ} \mathrm{C}$ for $10 \mathrm{~h}$, (b) $600{ }^{\circ} \mathrm{C}$ for $3 \mathrm{~h}$, and (c) $400^{\circ} \mathrm{C}$ for $10 \mathrm{~h}$. (d) Film deposited without atomic oxygen irradiation and crystallized by post-annealing at $400{ }^{\circ} \mathrm{C}$ for $10 \mathrm{~h}$.

to be formed or the chemical composition to be changed locally. The relationship between crystallization and annealing temperature was systematically studied by varying the temperature from 150 to $750^{\circ} \mathrm{C}$. The onset temperatures for random nucleation, growth, and continuous crystallization were evaluated by assessing the post-annealing temperatures of films deposited with and without irradiation.

Note that no XRD peak was detected for films deposited without irradiation for post-annealing temperatures lower than $350^{\circ} \mathrm{C}$ for $10 \mathrm{~h}$ (data not shown); however, a peak corresponding to BZY(110) appeared upon post-annealing at $400{ }^{\circ} \mathrm{C}$ for $10 \mathrm{~h}$ [Fig. 5(d)], suggesting that random nucleation is thermally activated between 350 and $400{ }^{\circ} \mathrm{C}$ in this film. In contrast, films deposited with atomic oxygen irradiation did not show any XRD peak for BZY even with postannealing at $400{ }^{\circ} \mathrm{C}$ for $10 \mathrm{~h}$ [Fig. 5(c)]. Annealing at higher temperatures progressively caused the appearance of small XRD peaks for BZY(110) and (200), as shown in Fig. 5(b), which shows XRD peaks for post-annealing at $600^{\circ} \mathrm{C}$ for $3 \mathrm{~h}$. Annealing at higher temperatures progressively caused the appearance of small XRD peaks for BZY(110) and (200), as shown in Fig. 5(b), which shows XRD peaks for postannealing at $600^{\circ} \mathrm{C}$ for $3 \mathrm{~h}$, and the intensity of two peaks increased by post-annealing at $750{ }^{\circ} \mathrm{C}$ for $10 \mathrm{~h}$ [Fig. 5(a)]. This result indicates that continuous crystallization starts at approximately $600^{\circ} \mathrm{C}$, which is a significantly higher temperature than that for random nucleation and growth. These systematic studies reveal that the temperature at which continuous epitaxial crystallization starts is still higher than the temperature that activates random nucleation, contrary to our hypothesis.

Cross-sectional TEM images of post-annealed specimens were examined to identify the reason for this disagreement. The cross-sectional micrographs of the film deposited without irradiation and post-annealed at $400{ }^{\circ} \mathrm{C}$ for $10 \mathrm{~h}$ [Fig. 6(a)] show small crystals measuring several tens of nanometers in the middle and upper portions of the BZY film. XRD and EBSD patterns indicate that these small crystallites originate from random nucleation and growth. Remarkably, no crystallite was visible at approximately $30 \mathrm{~nm}$ above the filminterlayer interface, and the density of this polycrystalline
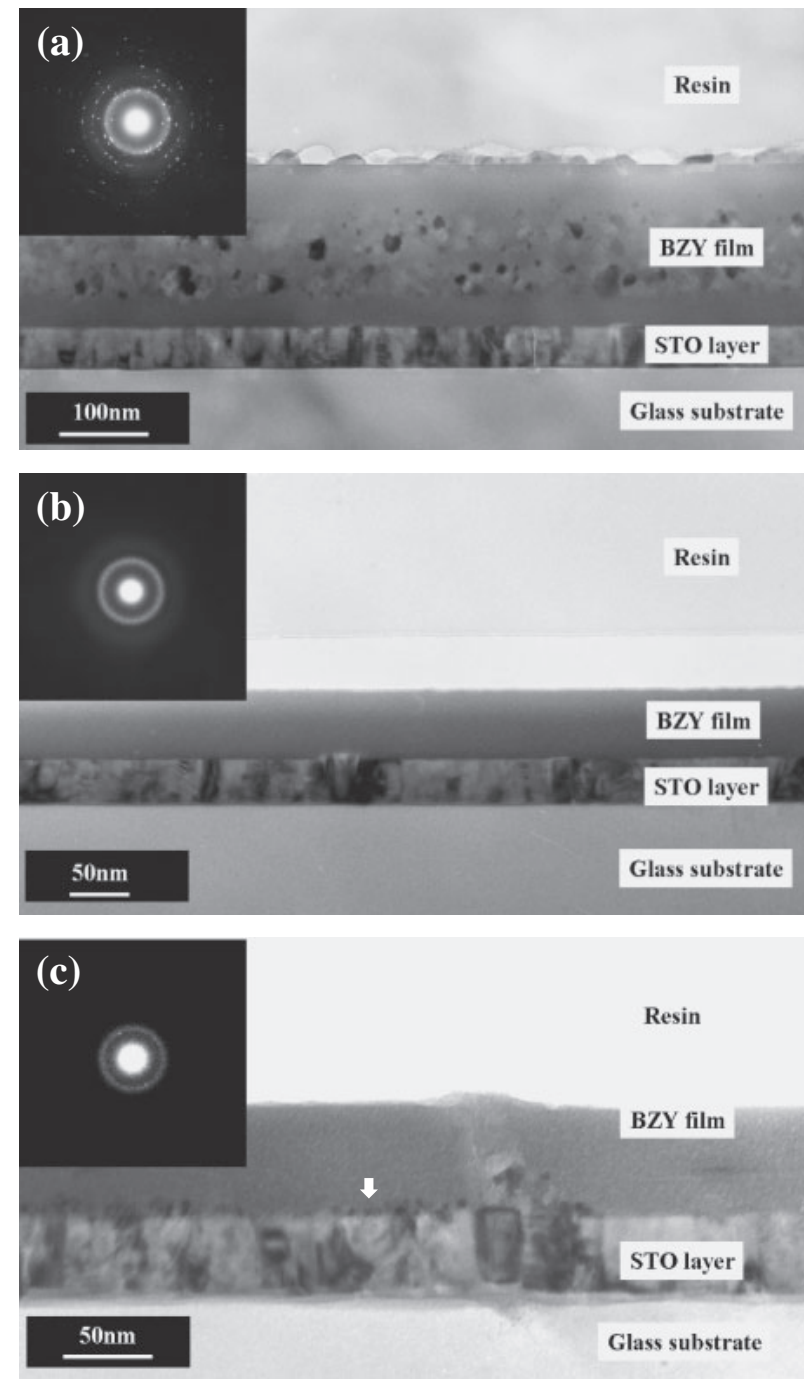

Fig. 6. Cross-sectional TEM images of BZY thin films crystallized under different post-annealing conditions. (a) Film deposited without atomic oxygen irradiation and crystallized by post-annealing at $400^{\circ} \mathrm{C}$ for $10 \mathrm{~h}$. Films deposited under atomic oxygen irradiation and crystallized by postannealing (b) at $400^{\circ} \mathrm{C}$ for $10 \mathrm{~h}$ and (c) $600{ }^{\circ} \mathrm{C}$ for $3 \mathrm{~h}$. Insets pictures show the EBSD patterns and white arrow indicates small grains.

layer seemed to decrease toward the surface. Moreover, no evidence for either random nucleation and growth or continuous crystallization was observed in the film deposited under irradiation and post-annealed at $400^{\circ} \mathrm{C}$ [Fig. 6(b)], consistent with the XRD results. Upon post-annealing at $600{ }^{\circ} \mathrm{C}$ for $3 \mathrm{~h}$, a columnar crystalline are observed, and small grains, which indicated by white arrow, appeared at the filminterlayer interface [Fig. 6(c)]. The inset EBSD pattern, which included diffraction from the film and a few small grains, suggested that the existence of amorphous phase and crystalline phase. Therefore, small grains confirmed to be crystallized BZY. This cross-sectional analysis agrees with the XRD result shown in Fig. 5(b), and revealed the starting points of continuous crystallization.

In summary, random nucleation and growth occurs between 350 and $400^{\circ} \mathrm{C}$ in films deposited without atomic oxygen irradiation, while continuous crystallization starts at approximately $600^{\circ} \mathrm{C}$ in films deposited under irradiation. The cross-sectional TEM image in Fig. 3(a) suggests that the polycrystalline area is produced first, and the columnar 
structure grows from the interface when the post-annealing temperature increases. On the other hand, random nucleation and growth does not occur above $400{ }^{\circ} \mathrm{C}$ in films deposited with irradiation. This exclusion of polycrystallinity promotes continuous crystallization from the film-interlayer interface and allows the columnar structure to reach the film surface [Fig. 3(b)]. These observations indicate that atomic oxygen irradiation during deposition prevents random nucleation and growth but does not facilitate continuous crystallization. Moreover, although the atomic oxygen only irradiated during the first $3 \%$ of the total film deposition, it impacted crystallization in the middle of the film.

Generally, the nucleation rate in glass is described by parameters of thermodynamics such as temperature, enthalpy of fusion, activation energy for diffusion, and interface energy between solid and amorphous phases, and it decreases as the interface energy and activation energy increase. ${ }^{31}$ ) Hence, either or both parameters must be different in the middle of the films when there is atomic oxygen irradiation in the early stages of film deposition.

Considering the properties of atomic oxygen and its configuration, as shown in Fig. 2, we do not believe that there is implantation of oxygen into the STO interlayer with irradiation, thereby changing the STO surface morphology, because the kinetic energy of the atomic oxygen is not sufficient. Highly reactive atomic oxygen would be adsorbed on the surface of the STO interlayer before deposition and the new BZY surface after each deposition pulse. Some of the adsorbed oxygen is introduced into the BZY films, because the temperature during deposition is low and does not allow the excess oxygen to diffuse outside of the film. This leads to local deformation, morphology changes, and chemical composition fluctuation around the interface region, as shown in Fig. 1. Finally, these changes are speculated to remain constant during deposition without relaxation, and they influence interface energy and activation energy for diffusion.

Through this study, we have speculated about the role of atomic oxygen irradiation, which changes the surface properties of BZY films and influences thermodynamic parameters in BZY films when coupled with low substrate temperature. Note that these speculations should be investigated in future experiments to gain further insight into the role of atomic oxygen irradiation. However, we believe that low temperature atomic oxygen irradiation could influence thermodynamic properties such as interface energy and activation energy for diffusion. Further elucidation of its role could lead to improvements in film growth with applications for improvement of solid-state electrochemical devices in future.

\section{Conclusions}

The crystallization of amorphous BZY thin films by postannealing was evaluated to understand the impact of atomic oxygen irradiation during deposition. Films deposited without irradiation on $\mathrm{STO} / \mathrm{SiO}_{2}$ were polycrystalline, because random nucleation and growth occurs at temperatures lower than the onset temperature for continuous crystallization at the film-interlayer interface. In contrast, films deposited under irradiation did not show random nucleation and growth at the high temperatures at which continuous crystallization starts. Therefore, although only applied near the filminterlayer interface, atomic oxygen irradiation during deposition prevents random nucleation in the middle of the thin films. We speculate that the change of surface properties by irradiation influences thermodynamic properties in the entire film.

\section{Acknowledgment}

This study was partly supported by a Grant-in-Aid for Young Scientists (A) 23686020 and a Grant-in-Aid for Scientific Research (A) 23246020 from the Japan Society for the Promotion of Science.

1) Y. P. Chao, W. Tang, and X. H. Wang, J. Mater. Sci. Technol. 28, 325 (2012).

2) Y. H. Hwang, H. M. Kim, Y. H. Um, and H. Y. Park, Mater. Res. Bull. 47, 2898 (2012)

3) O. Unal, T. E. Mitchell, and A. H. Heuer, J. Am. Ceram. Soc. 77, 984 (1994).

4) R. A. Roy, P. Catania, K. L. Saenger, J. J. Cuomo, and R. L. Lossy, J. Vac. Sci. Technol. B 11, 1921 (1993).

5) A. Ramalho, S. Fayeulle, and M. T. Vieira, Thin Solid Films 254, 131 (1995).

6) J. P. Nabot, B. Andre, and J. P. Chabert, Surf. Coatings Technol. 45, 359 (1991).

7) E. W. Kreutz, M. Alunovic, A. Voss, W. Pfleging, H. Sung, and D. A. Wesner, Surf. Coatings Technol. 68-69, 238 (1994).

8) D. Hondo, T. Tsurui, N. Kuwata, N. Sata, F. Iguchi, and H. Yugami, Solid State Ionics 178, 685 (2007).

9) N. Kuwata, N. Sata, T. Tsurui, and H. Yugami, Jpn. J. Appl. Phys. 44, 8613 (2005).

10) N. Kuwata, N. Iwagami, Y. Tanji, Y. Matsuda, and J. Kawamura, J. Electrochem. Soc. 157, A521 (2010).

11) N. Sata, K. Eberman, K. Eberl, and J. Maler, Nature 408, 946 (2000).

12) P.-C. Su, C.-C. Chao, J. H. Shim, R. Fasching, and F. B. Prinz, Nano Lett. 8, 2289 (2008)

13) M. Sase, F. Hermes, K. Yashiro, K. Sato, J. Mizusaki, T. Kawada, N. Sakai, and H. Yokokawa, J. Electrochem. Soc. 155, B793 (2008).

14) D. Pergolesi, E. Fabbri, A. D'Epifanio, E. Di Bartolomeo, A. Tebano, S. Sanna, S. Licoccia, G. Balestrino, and E. Traversa, Nat. Mater. 9, 846 (2010).

15) T. Ishihara, H. Eto, and J. W. Yan, Int. J. Hydrogen Energy 36, 1862 (2011).

16) H. Yugami, F. Iguchi, and H. Naito, Solid State Ionics 136-137, 203 (2000).

17) X. D. Wu, R. E. Muenchausen, N. S. Nogar, A. Pique, R. Edwards, B. Wilkens, T. S. Ravi, D. M. Hwang, and C. Y. Chen, Appl. Phys. Lett. 58, 304 (1991).

18) F. Iguchi, T. Tsurui, N. Sata, and H. Yugami, Hyomen Kagaku 29, 396 (2008) [in Japanese].

19) P. de Coux, R. Bachelet, C. Gatel, B. Warot-Fonrose, J. Fontcuberta, and F. Sánchez, CrystEngComm 14, 7851 (2012).

20) A. Yamada, K. Saka, M. Uehara, S. Taminato, R. Kanno, F. Mauvy, and C. Grenier, Electrochem. Commun. 12, 1690 (2010).

21) D. Mori, H. Oka, Y. Suzuki, N. Sonoyama, A. Yamada, R. Kanno, Y. Sumiya, N. Imanishi, and Y. Takeda, Solid State Ionics 177, 535 (2006).

22) J. Garcia-Barriocanal, A. Rivera-Calzada, M. Varela, Z. Sefrioui, E. Iborra, C. Leon, S. J. Pennycook, and J. Santamaria, Science 321, 676 (2008).

23) M. N. K. Bhuiyan, H. Kimura, T. Tambo, and C. Tatsuyama, Jpn. J. Appl. Phys. 43, 7879 (2004).

24) H. Ohta, J. Ceram. Soc. Jpn. 114, 147 (2006).

25) H.-G. Chen and Y.-S. Lin, Thin Solid Films 545, 33 (2013).

26) Y. Nakamura, S. Kudo, M. Mukaida, and S. Ohshima, Physica C 378-381, 1241 (2002).

27) N. Sata, presented at 15th Int. Conf. Solid State Protonic Conductors, 2010.

28) G. Koren, A. Gupta, and R. J. Baseman, Appl. Phys. Lett. 54, 1920 (1989).

29) W. Jo, L. S. J. Peng, W. Wang, T. Ohnishi, A. F. Marshall, R. H. Hammond, M. R. Beasley, and E. J. Peterson, J. Cryst. Growth 225, 183 (2001).

30) N. Sata, H. Matsuta, Y. Akiyama, Y. Chiba, S. Shin, and M. Ishigame, Solid State Ionics 97, 437 (1997).

31) S. Sakka, Garasu Kagaku no Kiso to Oyo (Uchida Rokakuho, Tokyo, 1997) p. 89 [in Japanese]. 